\title{
The Effects of Xylitol on the Secretion of Insulin and Gastric Inhibitory Polypeptide in Man and Rats
}

\author{
S. Salminen, E. Salminen and V. Marks \\ Department of Biochemistry, Division of Clinical Biochemistry, University of Surrey, Guildford, UK
}

Summary. Xylitol given by gavage to unadapted rats produced profound diarrhoea and no rise in plasma gastric inhibitory polypeptide (GIP) concentration. In xylitol adapted rats it did not cause diarrhoea but still had no effect upon GIP release in contrast to glucose, which did. In healthy human subjects xylitol taken by mouth in solution as a single $30 \mathrm{~g}$ dose produced only a minimal rise in blood glucose and no rise in plasma GIP or insulin concentration. Glucose in similar doses, on the other hand, caused a large rise in all three.

Key words: Xylitol, GIP, insulin, diarrhoea, rat, man.

There has been considerable interest of late in the possibility of using unabsorbable $[1,2]$ or slowly absorbed $[3,4]$ carbohydrates in the dietary management of diabetes. Xylitol, a sweet five-carbon sugar alcohol, thought to be absorbed slowly by passive diffusion, has been proposed as a potential sugar substitute. It is also thought to increase gastrointestinal motility in man [3] but its effect on the secretion of GIP has not yet been reported. We examined both the GIP and insulinotropic properties of xylitol in human volunteers and compared them with results obtained in rats which had, or had not been adapted to dietary xylitol.

\section{Materials and Methods}

\section{Animal Study}

Male Wistar albino rats weighing between $220-250 \mathrm{~g}$ were divided into three groups. One group served as the controls and received oral glucose in the test; another received xylitol in the test but with- out adaptation by prior introduction of xylitol into the diet, whilst the third group was adapted, by gradually increasing the xylitol content of the diet to a maximum of $20 \%$ [4], before receiving oral xylitol in the test. The animals were starved for $24 \mathrm{~h}$ before being given either xylitol or glucose $(1.2 \mathrm{~g} / \mathrm{kg}$ body weight $)$ as a $25 \%$ solution by stomach tube. Blood was collected from the tail vein into heparinised tubes at $0,15,30,60$ and $120 \mathrm{~min}$ and separated immediately by centrifugation at $2000 \times g$, for subsequent measurement of plasma immunoreactive GIP by a double-antibody radioimmunoassay technique [5].

\section{Human Volunteer Study}

The study group consisted of six healthy volunteers (three males and three females) aged 26-36 years, none of whom had been introduced to xylitol before the study. On each of two occasions, after a $12 \mathrm{~h}$ overnight fast, the volunteers drank a test solution containing either $30 \mathrm{~g}$ of glucose or $30 \mathrm{~g}$ of xylitol dissolved in $200 \mathrm{ml}$ of lemonflavoured water. The test solutions were taken one week apart in random order and were consumed within $5 \mathrm{~min}$.

Venous blood was collected through an indwelling cannula before and at frequent intervals for $120 \mathrm{~min}$ after starting the test. An aliquot was analysed for glucose using an automated glucose-oxidase method. The remainder was separated by centrifugation, the plasma separated and analysed for immunoreactive insulin [6] and immunoreactive GIP [5]. The results were compared using Student's two-tailed ' $t$ ' test.

\section{Results}

\section{Animal Studies}

Figure 1 compares the mean GIP responses to glucose and xylitol in Wistar rats that had, or had not, been adapted to a high xylitol intake. Glucose administration caused a significant increase in GIP secretion $(p<0.01)$ whereas xylitol administration did not do so, regardless of whether adaptation had occurred or not. Rats that were not adapted had diarrhoea about $1 \mathrm{~h}$ after administration of the test dose of xylitol; this was not observed in rats in the xylitol-adapted group, either during or after the test. 


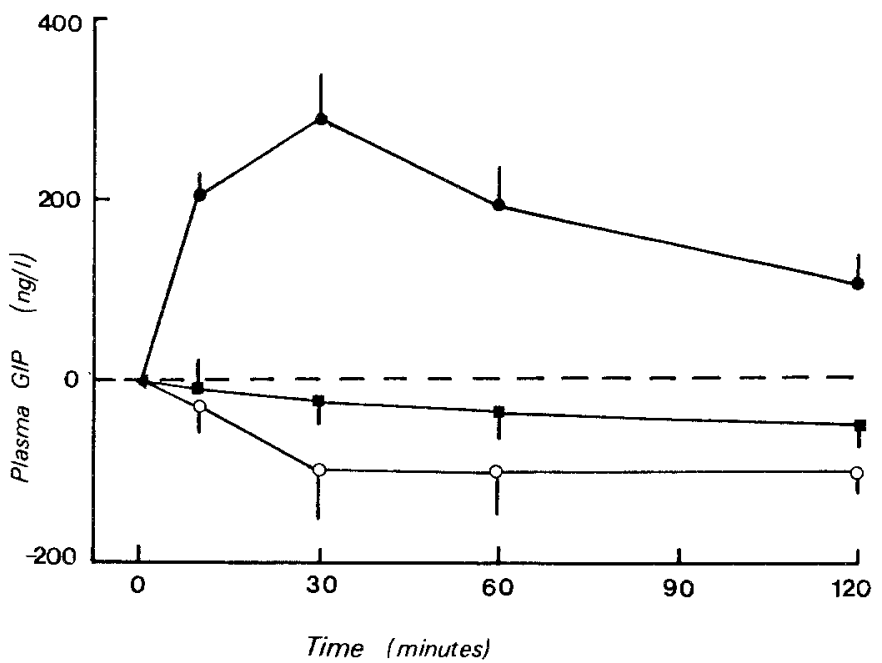

Fig.1. Mean GIP responses in Wistar rats after oral administration of glucose, xylitol without adaptation or xylitol with adaptation, at $1.2 \mathrm{~g} / \mathrm{kg}$ body weight. Each value is the mean \pm SEM for five rats $(\bullet=$ glucose, $O=$ xylitol without prior adaptation, $\boldsymbol{\square}=$ xylitol with adaptation)

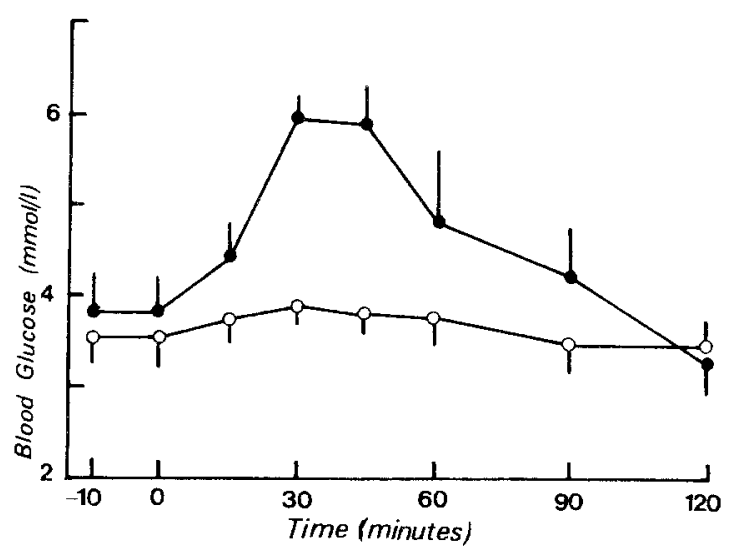

Fig. 2. Mean \pm SEM blood glucose levels in six normal subjects given $30 \mathrm{~g}$ xylitol or glucose by mouth $(\mathrm{O}=$ xylitol, $=$ glucose $)$

\section{Human Studies}

Figure 2 compares the changes in mean blood glucose concentration in the volunteers after glucose and xylitol administration. As expected, oral glucose caused a marked rise $(2.5 \pm 0.4 \mathrm{mmol} / \mathrm{l} ; p<0.01)$ in mean blood glucose concentration which had returned to slightly below the basal level by the end of the test. Xylitol produced only a small, but significant, rise in mean blood glucose concentration $(p<0.05)$ which remained above fasting levels throughout the period of observation.

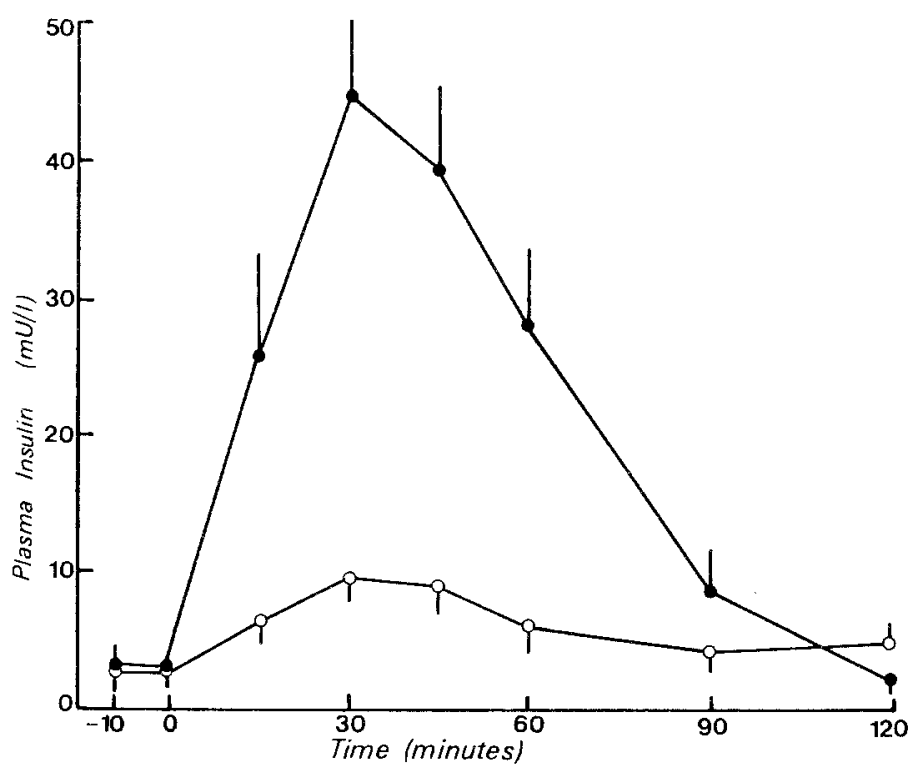

Fig.3. The effect of xylitol and glucose on plasma insulin in six normal subjects during oral carbohydrate loading tests. Each value is the mean \pm SEM for six subjects $(O=$ xylitol, $\bullet=$ glucose $)$

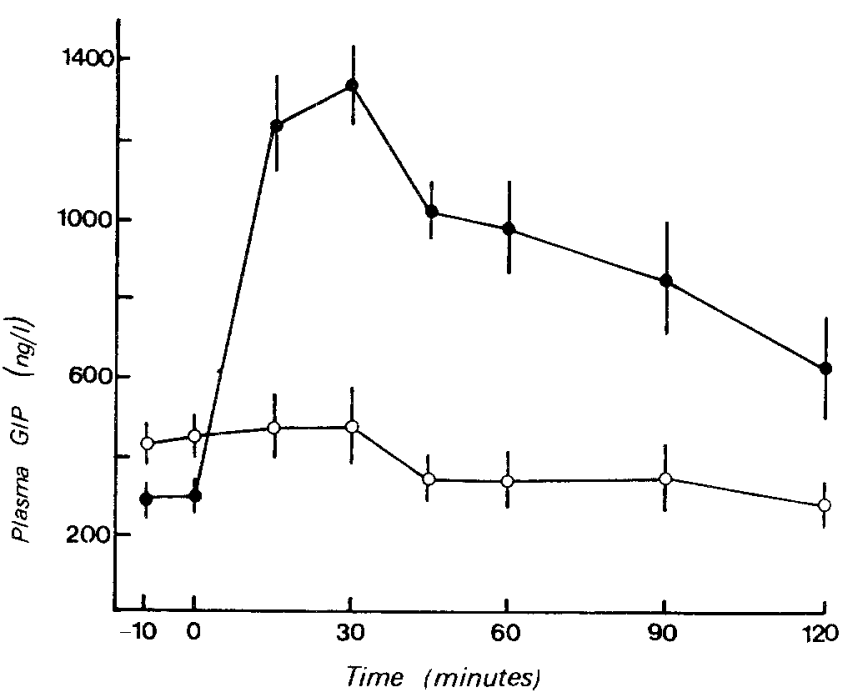

Fig.4. The effect of xylitol and glucose on secretion of GIP during oral carbohydrate loading tests in human volunteers. Each value is the mean \pm SEM for six subjects $(\mathrm{O}=$ xylitol, $\boldsymbol{0}$ = glucose $)$

Plasma immunoreactive insulin levels rose significantly after both sugars, though considerably more so after glucose than after xylitol (Fig.3). Secretion of immunoreactive GIP was significantly increased by glucose $(p<0.01)$ but not by xylitol administration; indeed, mean plasma GIP levels decreased during the course of the study (Fig. 4).

Two of the volunteers experienced transient diarrhoea after drinking the xylitol solution and one complained of flatulence. None reported any side-effects after the administration of the glucose solution. 


\section{Discussion}

The GIP responses to oral glucose observed during this study are similar to those previously observed in rats [7] and man [1]. Xylitol did not stimulate GIP secretion in either species. Even long-term adaptation did not reverse the lack of GIP-response to xylitol although its absorption was apparently improved.

Earlier studies have shown that GIP secretion is stimulated by the ingestion of glucose or triglyceride and that the release of GIP from the gut in response to glucose might be regulated by the rate of absorption. The molecular configuration of monosaccharides with the ability to stimulate GIP release has been shown to agree well with the requirements for active transport by the sodium-dependent hexose pathway [8]. Xylitol differs in structure from the common actively-transported sugars and is thought to be absorbed by passive diffusion [3]. The results in rats indicate that, even though an absorptive adaptation occurs when large doses of xylitol are consumed over prolonged periods, it appears not to be associated with the appearance of an active transport system.

Acknowledgements. We would like to thank the Finnish Academy for a scholarship for ES and Finnsugar for financial support.

\section{References}

1. Morgan LM, Goulder TJ, Tsiolakis D, Marks V, Alberti KGMM (1979) The effect of unabsorbable carbohydrate on gut hormones. Diabetologia 17: 85-89
2. Jenkins DJA, Leeds AR, Gassuyl MA, Crochet B, Alberti KGMM (1977) Decrease in post-prandial glucose concentrations by guar and pectin. Ann Intern Med 86:20-23

3. Förster H (1978) Tolerance in the human. Adults and children. In: Counsell JN (ed) Xylitol. Applied Science Publishers, London, pp 43-66

4. Bässler KH (1969) Absorption of xylitol. In: Horecker BL, Lang K, Takagi Y (eds) Pentoses and Pentitols. Springer Verlag, Berlin pp 190-195

5. Morgan LM, Morris BA, Marks V (1978) Radioimmunoassay of gastric inhibitory polypeptide. Ann Clin Biochem 15:172-177

6. Soeldner JS, Stone D (1965) Critical variables in the radioimmunoassay of serum insulin using a double antibody technic. Diabetes 14:771-779

7. Morgan LM (1979) Immunoassayable gastric inhibitory polypeptide: Investigations into its role in carbohydrate metabolism. Ann Clin Biochem 16: 6-14

8. Sykes S, Morgan LM, English J, Marks V (1980) Evidence for preferential stimulation of gastric inhibitory polypeptide secretion in the rat by actively transported carbohydrates and their analogues. J Endocrinol 85: 201-207

Received: 13 July 1981

and in revised form: 25 November 1981

Professor V. Marks

Department of Biochemistry

University of Surrey

Guildford

Surrey GU 25 XH, UK 